\title{
Association of COVID-19 and Cancer: Risk Statistics and Management of COVID-19 in Cancer Patients
}

\section{Shahzar Khan', Hasnain Jan²,3, Shah Faisal ${ }^{2}$, Ayyaz Khan ${ }^{3}$, Rashida llyas ${ }^{4}$, Khadija Bibi $^{4}$, Sadeeq ur Rehman ${ }^{4}$}

1. Department of Microbiology, Abdul Wali Khan University, Mardan, KPK, Pakistan.

2. Department of Biotechnology, Bacha Khan University, Charsadda, KPK, Pakistan.

3. Department of Biotechnology, Quaid-i-Azam University, Islamabad 45320, Pakistan

4. Department of Botany, Govt. Post Graduate College, Bacha Khan University, Charsadda, KPK, Pakistan.

\section{Corresponding author:}

Hasnain Jan

Email: hasnainjan@bs.qau.edu.pk

\begin{abstract}
COVID-19 is extremely lethal disease and almost 190 countries are suffering from the latest pandemic. The literature indicates that COVID-19 is more prevalent in patients with compromised immune systems. Patients with cancer are particularly vulnerable to COVID-19 because of their immune compromised condition due to immunosuppressive therapy. Immunosuppressive therapy results in mixing or overlap of COVID-19 associated pneumonia and immune-related pneumonitis and thus makes the diagnostic process very confusing. On the other side in this pandemic, treating cancer patients in hospital will bring a lot of risk. There is still no strong evidence on the cancer and COVID-19 connection. Yet, in this pandemic, patients with cancer should be treated as special cases. Risk management is highly needed in the critical time of this pandemic. This review highlights the association between COVID-19 and cancer, and we also highlighted the strategies which should be used to minimize the risk of COVID-19 in cancer patients.
\end{abstract}

Keywords: COVID-19; cancer, review

Citation: $\quad$ Khan S, Jan H, Faisal S, Khan A, llyas R, Bibi K, Rehman S. Association of COVID-19 and Cancer: Risk Statistics and Management of COVID-19 in Cancer Patients. International Cardiovascular Forum Journal 2020;20:5-9.DOI: 10.17987/icfj. v20i0.691

\section{Introduction}

In December 2019, an outbreak of novel corona virus (COVID-19) also known as (SARS-CoV-2) in Wuhan state, Hubei province of china was reported which then spreads to other regions of China [1-3]. Because of human-to-human transmission, the worldwide health organization has declared a pandemic spread worldwide [4]. Initial symptoms of 2019 novel coronavirus are fever accompanied by respiratory abnormalities like coughing and shortening of breath. Only 15 to $20 \%$ of the patients have these mild abnormalities while remaining asymptomatic in majority of the patients. Older age patients are more susceptible to this new corona virus [5]. Coronaviruses are enveloped viruses with positive strand RNA as described by Tyrell and Bynoe in 1996 [6]. They are spherical virions with surface projection and have four sub families alpha, beta, gamma and delta, and genome size range between $26 \mathrm{~kb}$ and $32 \mathrm{~kb}$. Their normal hosts are pigs and birds [7]. Although COVID-19 can infect everyone but patients with cancer are at high risk from this pandemic. Recently many patients with cancer have been diagnosed positive for COVID-19, approximately $5.6 \%$ cancer patients died of coronavirus which is very high as compared to $2.6 \%$ fatality rate of the non-cancer patients $[8,9]$. Due to release of the immunosuppressive cytokines in cancer patients which suppresses the leukocytes population meaning cancer patients become more prone to the COVID-19 [10, 11]. Smoking, a the major cause of the cancer also enhances the gene regulation and expression of Angiotensin converting enzyme 2 receptor the binding site for SARS-COV and thus increases the susceptibility to COVID-19 [12]. Liang and colleagues first reported COVID-19 to be more prevalent in cancer patients who become high risk therefore for intensive care [8]. Immunosuppressive therapy in cancer patients and it's association with COVID-19 should not be neglected [10]. This review is an effort to highlight the association of cancer and COVID-19 and risk statistics and risk management of COVID-19 in cancer patients, however, there are no clear evidences on association between COVID-19 and cancer therapy. 


\section{Anticancer therapy increasing the risk COVID-19 susceptibility in cancer patients}

Cancer patients are more prone to COVID-19 and they may develop severe and lethal complications [13]. Immune suppression due to chemotherapy favors more suitable environment for coronavirus infection and complication in cancer patients. Conversely, patients receiving immunotherapy should be more immune reactive. Deadly inflammation and anti-programmed cell death protein 1/ programmed death ligand-1 or anti-CTLA4 immune checkpoint inhibitors (ICls) result in lung injury mediated by a cytokine storms and may have a harmful effect on coexisting COVID-19. Cytokine storms are considered responsible for the above events and cytokine storm is a known complication of SARS-CoV-2 [14, 15]. This virus can also effect macrophages, $T$ cells and other immune cell which then interfere with the immunotherapy resulting in mixing or overlap of COVID-19 associated pneumonia and immune-related pneumonitis. These two disease have similarity in lot of their clinical and radiologic properties which can make the diagnosis process very confusing. On other hand immune-related pneumonitis can be treated by injecting corticosteroids through intravenous route but this has antagonistic role in the treatment of COVID-19 related pneumonia [16].

\section{Association of COVID-19 and chemotherapy in lung cancer patients}

Zhang et al., who was the first to report the association of the lung cancer and COVID-19. A Chinese man of 57 years age has lung adenocarcinoma and harboring EGFR L858R mutation, but he continues targeted therapy (osimertinib) against lung cancer and also having COVID-19 associated pneumonia. The patient then recovered from pneumonia and also, he had gained a stable control of the cancer. Here the author has admitted that the patient was taking anticancer therapy osimertinib and still recovered, but if we look at the clinical practice it may not be possible because there is a heterogeneous relation between lung cancer and COVID-19 and the symptoms may range from mild to very severe [17]. Tumors harboring EFGR mutation when treated with targeted therapy may affect young and never-smoker patients, who will shows minorities of these complications, in contrast older age people will be more affected by these situations. Because they will go for chemotherapy, immunotherapy, and not targeted therapy therefore COVID-19 may be more severe in patients with older age and tumor. In this context, patients have a higher risk of developing severe or lethal SARS-CoV-2 complications. In addition, there is an increasing debate on potential interactions between corona virus and anticancer therapies [13].

\section{Differential diagnoses of COVID-19 pneumonia in lung cancer patients}

COVID-19 associated pneumonia is very different from chronic pneumonia and other infections as confirmed from the images from CT scan, epidemiological study and other diagnostic tests. There is a lot of genetic similarity between SARS-COV and Covid-19. Recently it has been renamed by International Committee on Taxonomy of Viruses as SARS-COV-19. Comparing the pathological similarities SAR-CoV-2 has same complexity of acute lung injury, phases of acute lung injury, such as edema, inflammatory infiltrate, type II pneumocyte hyperplasia, and organization, but without obvious hyaline membrane formation and other long-term processes, such as squamous metaplasia $[18,19]$.
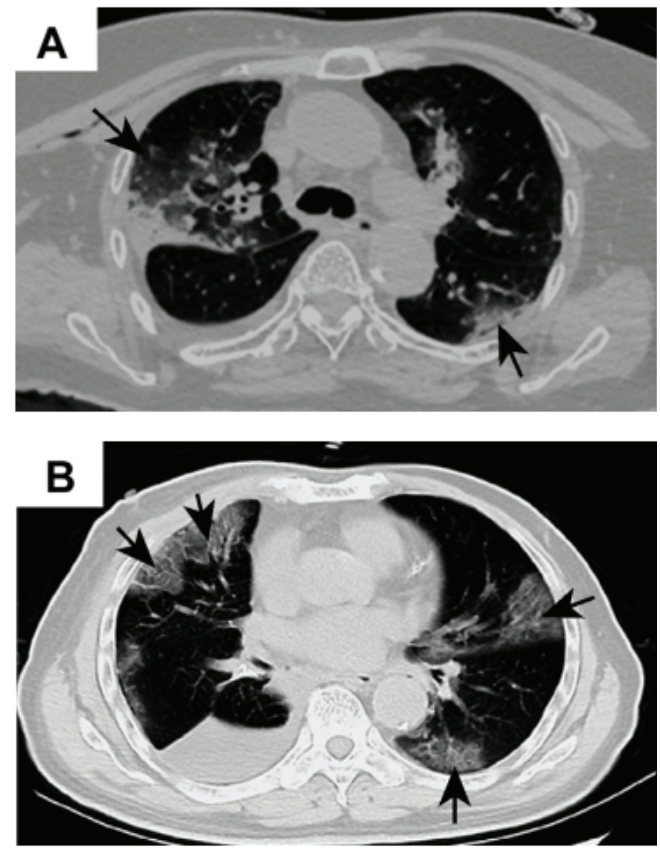

Figure. 1. Adapted from [20] shows the CT scan image of the lung cancer patients being positive for the COVID -19. (A) Represents the post-operative image of the right lung revealing changes in the right lung and increased ground-glass opacities bilaterally (arrows). (B) Foci of ground-glass opacity seen bilaterally (arrows).

Histological changes of early phase COVID-19 Pneumonia in Two Patients with Lung Cancer

Pathologic findings from two patients were edema and prominent proteinaceous exudates, vascular congestion, and inflammatory clusters with fibrinoid material and multinucleated giant cells [20]

\section{Evolution of COVID-19 in patient taking a long course of anticancer therapy}

A 65 years old male patient was admitted in emergency department for shortness of breath, fever, and mental confusion. When the hematological analysis was done, he showed hypoxia and lymphopenia, his C-reactive protein level was highly elevated and had also high levels of transaminases and lactate dehydrogenase. Reticular-interstitial adversative was found on performing the Chest $\mathrm{x}$-ray. By performing RT-PCR from nasal swab he was positive for COVID-19. On studying his medical history, he was found positive for emphysema and lung adenocarcinoma which was diagnosed on August 2012. For the cancer treatment he had undergone cerebral metastasectomy and radiotherapy. After six cycles of chemotherapy brain MRI and CT scan showed progression of disease. He was then enrolled in CA209-057 clinical trial and treated, from August 2013 until February 14, 2020, with nivolumab, a PD-1 checkpoint inhibitor, without adverse event and with a hold partial response. The last CT scan was performed on February 2, 2020 and described stable disease. On March 5, 2020, he was admitted in infectious disease unit and started empiric antibiotic treatment and oxygen therapy with reservoir mask at $15 \mathrm{~L} / \mathrm{min}$. He was sedated for agitation and, for this reason, he never received prescribed lopinavir + ritonavir and hydroxychloroquine. The patient had a rapid worsening of condition and died on March 9 [21]. 


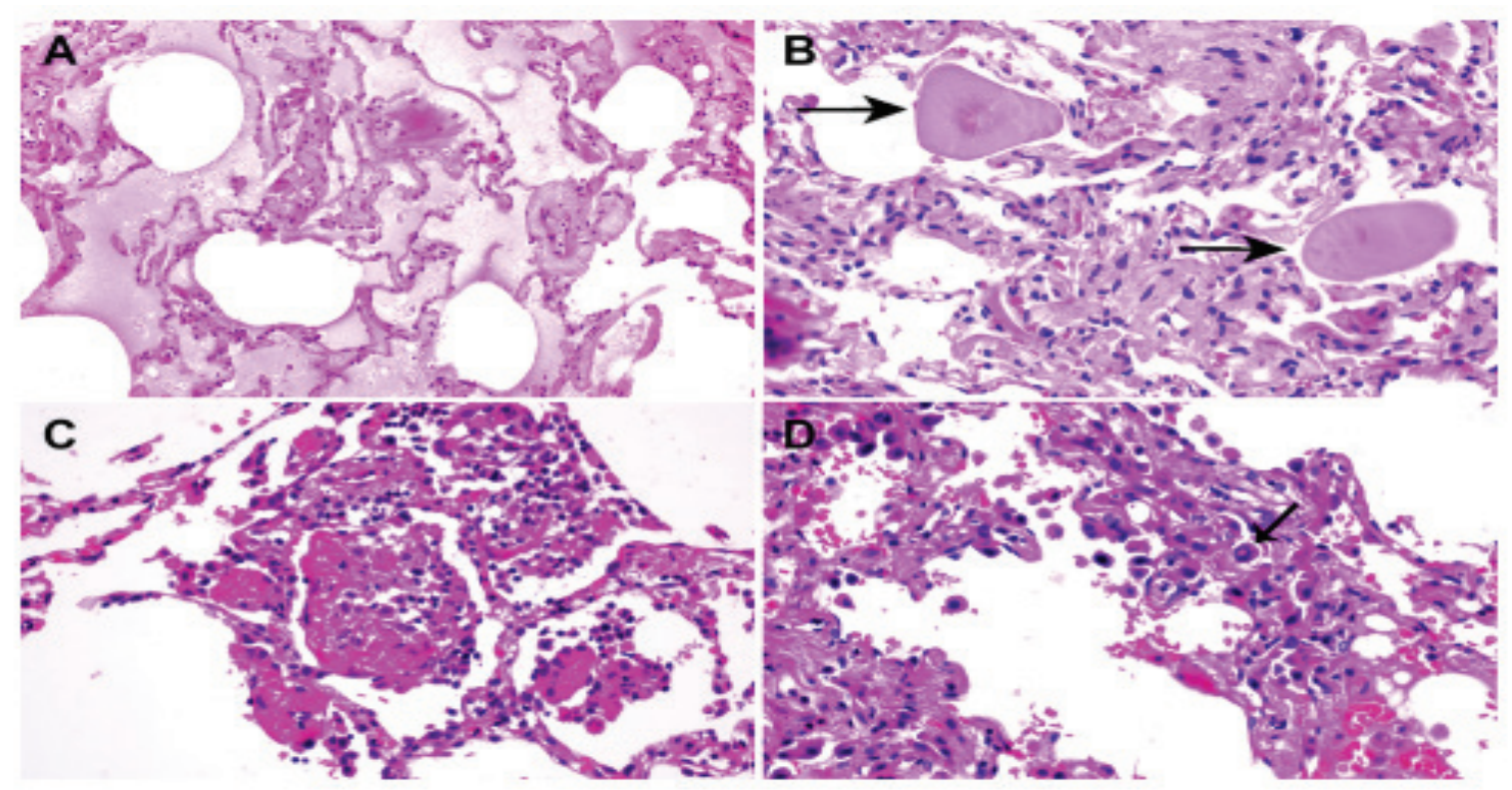

Figure 2. Adapted from [20] shows histological changes from case 1. (A) Proteinaceous exudates in alveolar spaces, with granules; (B) scattered large protein globules (arrows); (C) intra-alveolar fibrin with early organization, mononuclear inflammatory cells, and multinucleated giant cells; (D) hyperplastic pneumocytes, some with suspected viral inclusions (arrow).

\section{Case fatality rate of COVID-19 patients with cancer and other co-morbidities}

Overall case fatality rates of COVID-19 recorded in China with cancer and other co-morbidities was $2.3 \%$ compared with $7.2 \%$ in Italy. On comparison the CFR of China and Italy shows that CFR shows similarity in patients with age less than 70 years, but it is higher in Italy for older age above 70 years. The percent CFR recorded above age 90 years was $37.6 \%$ in Italy and $11.9 \%$ in China. Although the overall CFR difference is also on the basis of different testing technology in these countries as Italy first adapted a testing strategy for both symptomatic and non-symptomatic patients but when patients suffered from severe SARS-CoV-2-related ARDS, the Italian Ministry of Health decided to allow testing only in symptomatic patients who were potential candidates for hospitalization, and this decision may have resulted in a biased selection and delayed treatment of these patients. In this editorial, we would like stress the identification of lung cancer patients as a specific population for testing prioritization for COVID-19 [22, 23].

\section{Risk Statistics of COVID-19 in cancer patients taking immunosuppressive therapy}

In the Lancet Oncology on Feb 14, 2020 Wenhua Liang and colleagues published a comment which focuses on the risk of COVID-19 in cancer patients. Using epidemiological statistics, the authors concluded that cancer patients have more chances to develop COVID-19. They studied the association of cancer and COVID-19 in 18 patients who have history of cancer among 1590 patients found positive for COVID-19 and having no history of cancer. Of 18 patients 4 had undergone chemotherapy, 12 patients had recovered from cancer through surgery or chemotherapy, and two had unknown treatment status. All were found positive for COVID-19 [8, 24]. Zhang et al., 2020 conducted study on 28 cancer patients for quarantine and treatment against COVID-19 having lung cancer, esophageal cancer, and breast cancer. Six patients out of 28 patients had received immunosuppressive
Table 1. Shows the CFR of COVID-19 in China and Italy patients with cancer and other co-morbidities.

\begin{tabular}{|c|c|c|c|}
\hline $\begin{array}{l}\text { Case } \\
\text { fatality rate }\end{array}$ & Age & $\%$ CFR & Co-morbidities \\
\hline China & Less than 70 years & $2.3 \%$ & diabetes \\
\hline Italy & Less than 70 years & $7.2 \%$ & $\begin{array}{l}\text { Cardiovascular } \\
\text { disease }\end{array}$ \\
\hline China & More than 70 years & $11.9 \%$ & cancer \\
\hline Italy & More than 70 years & $37.6 \%$ & $\begin{array}{l}\text { Chronic respiratory } \\
\text { disease }\end{array}$ \\
\hline
\end{tabular}

Table 2. Shows Comparison of risk of covid-19 in cancer patients and with patients with other co-morbidities [reproduced from WHO, 2020, February 28].

\begin{tabular}{|l|l|}
\hline CO-MORBIDITIES & $\%$ RISK \\
\hline CARDIOVASCULAR DISEASE & $13 \%$ \\
\hline DIABETUS & $9.2 \%$ \\
\hline HYPERTENSION & $8.4 \%$ \\
\hline CHRONIC RESPIRATORY DISEASE & $8.0 \%$ \\
\hline CANCER & $7.6 \%$ \\
\hline
\end{tabular}

therapy, antitumor therapy, radiation therapy 14 days before diagnosis and had developed severe events $(\mathrm{HR}=4.079,95 \% \mathrm{Cl}$ 1.1 - 15.3, $\mathrm{P}=0.037)$ ) and were more susceptible to COVID-19. Out of 28 patients $53.6 \%$ develop severe symptoms, $21.4 \%$ were admitted to ICU, 35.7\% had developed severe complications, and $28.6 \%$ had died [17]. 


\section{COVID-19 Risk assessment and risk management in cancer patients}

The following precautions should be adopted to minimize the risk of COVID-19 in cancer patients.

\section{Minimizing the risk of COVID-19 in cancer patients}

Corona virus can be spread by physical contacts and other ways but in case of cancer treatment we need an alternative to combat the high risk of COVID-19 in cancer patients through telephone/ telemedicine against multiple cancer types, such as endometrial [25], prostate [26] lung [27] and colorectal cancer, which may be assessed and treated in patients with cancer and other co-morbidities [28].

\section{Management of head and neck cancer care in COVID-19 pandemic situation}

Head and neck cancer is a rare form of cancer in Italy with 9300 cases and 3216 deaths. HNC is usually treated at very advanced stage of the diagnosis and needs rapid treatment, however treating $\mathrm{HNC}$ at this pandemic situation would be problematic. There should be a proper risk assessment team for the HNC patients who will weigh the benefits and risk of the treatment of the patients. A proper and reasonable treatment strategy for anticancer and epidemic prevention should be adopted i.e. avoid systemic therapy in patient having age more than 70 years and with major co-morbidities. These co-morbidities are increasing the risk of COVID-19. While in head and neck cancer when the doctors are monitoring the course of the COVID-19 patients the anticancer therapy should be delayed [29-32]

\section{Management of the radiation oncology clinic in COVID-19 pandemics}

Radiation oncologist should also stop the radiotherapy in cancer patients testing positive for the COVID-19, because $6.1 \%$ of COVID-19 patients with pneumonia and lymphocytopenia $91.1 \%$ and $83.2 \%$, respectively require intensive care [33]

\section{Risk management in colorectal cancer patients}

In patients with colorectal cancer, survival is lower if treatment is started $>90$ days from diagnosis. The same is the case with colon cancer patients when treatment is started after 3-6 months of diagnosis; however, avoiding delay in this pandemic is problematic. Oncologists should delay treatment for the good of the patient in this pandemic [34-36].

- According to American society of cancer there would 147,950 new collateral cancer in US.

- With $40 \%$ have chances of local diseases.

- 24,650 patients could be affected in 2 months of epidemic in the United States alone, of whom $\approx 9860$ have localized disease [37].

\section{Critical recommendations to oncologist by the Italian Association of Medical Oncology in COVID-19 pandemics}

The Italian Association of Medical Oncology (AIOM) in partnership with the boards of Academic Oncologists (COMU) and of Oncology Unit Directors (CIPOMO) proposes some recommendations for the follow up patients (patients who are taking active treatment) and for their care taker and these are: (1) Immediate delay of the anticancer therapy while observing the infections of SARSCoV-2. (2) For follow up patient the oncologist should avoid the disease-free patient from routine visit to the hospital; a phone call discussion of clinical documentation may be useful in this regard. (3) For admission to the hospital the patient should come alone $[18,38]$.

\section{Conclusion}

It is important to remember that covid-19 is highly infectious. And the patients who are already immune compromised or taking immunosuppressive therapy are highly prone to COVID-19. Cancer patients are highly vulnerable to COVID-19 because of the immunosuppressive therapy, targeted therapy and radiotherapy. There are a lot of challenges regarding the management of cancer patients in the COVID-19 pandemic. But there is a need of managing the risk of COVID-19 in cancer patients. In this regard the Italian Association of Medical Oncology (AIOM) proposes some recommendations for the follow up patients (patients who are taking active treatment); to delay immunosuppressive therapy in pandemic, consult patients through video call or phone call to avoid human to human transmission of COVID-19. However, there is no clear evidence on the association between cancer and COVID-19. But management of COVID-19 risk in cancer patients is highly needed because cancer patients are highly prone to COVID-19 pandemic.

\section{Conflict of interest}

The authors declare no conflicts of interest.

\section{Acknowledgements}

The authors state that they abide by the "Requirements for Ethical Publishing in Biomedical Journals" [39].

\section{References}

1. Huang $\mathrm{C}$ et al. Clinical features of patients infected with 2019 novel coronavirus in Wuhan, China. Lancet, 2020; 395(10223):497-506. doi. org/10.1016/S0140-6736(20)30183-5

2. Zhu $\mathrm{N}$ et al. A novel coronavirus from patients with pneumonia in China, 2019. New England Journal of Medicine, 2020. doi: 10.1056/ NEJMoa2001017

3. Jan $\mathrm{H}$ et al. COVID-19: Review of Epidemiology and Potential Treatments Against 2019 Novel Coronavirus. Discoveries, 2020. 8(2). doi: 10 . $15190 /$ d.2020.5

4. Baloch S et al. The Coronavirus Disease 2019 (COVID-19) Pandemic. The Tohoku Journal of Experimental Medicine, 2020. 250(4):271-278. doi. org/10. 1620/tjem.250.271

5. Bai, $Y$ et al. Presumed asymptomatic carrier transmission of COVID-19. Jama, 2020. 323(14):1406-1407. doi:10.1001/jama.2020.2565

6. Tyrrell $D$ and $M$ Bynoe. Cultivation of viruses from a high proportion of patients with colds. Lancet, 1966.

7. Lillie PJ et al. Novel coronavirus disease (Covid-19): the first two patients in the UK with person to person transmission. Journal of Infection, 2020. 80(5):578-606. doi.org/10.1016/j.jinf.2020.02.020

8. Liang $W$ et al. Cancer patients in SARS-CoV-2 infection: a nationwide analysis in China. The Lancet Oncology, 2020. 21(3):335-337. doi. org/10.1016/S1470-2045(20)30096-6

9. Yu J et al. SARS-CoV-2 transmission in cancer patients of a tertiary hospital in Wuhan. medRxiv, 2020. doi.org/10.1101/2020.02.22.20025320

10. Xia Y et al. Risk of COVID-19 for patients with cancer. The Lancet Oncology, 2020. 21(4):180. doi.org/10.1016/S1470-2045(20)30150-9

11. Picchianti DA et al. Cytokine Release Syndrome in COVID-19 Patients, A New Scenario for an Old Concern: The Fragile Balance between Infections and Autoimmunity. International Journal of Molecular Sciences, 2020. 21(9):3330. doi.org/10.3390/ijms21093330

12. Smith JC and Sheltzer JM. Cigarette smoke triggers the expansion of a subpopulation of respiratory epithelial cells that express the SARS-CoV-2 receptor ACE2. bioRxiv, 2020. doi.org/10.1101/2020.03.28.013672

13. Bersanelli M. Controversies about COVID-19 and anticancer treatment with immune checkpoint inhibitors. Future Medicine. 2020. doi.org/10.2217/imt2020-0067

14. Mehta $P$ et al. COVID-19: consider cytokine storm syndromes and immunosuppression. The Lancet, 2020. 395(10229):1033-1034. doi. org/10.1016/S0140-6736(20)30628-0 
15. Postow MA, Sidlow R, and Hellmann MD. Immune-related adverse events associated with immune checkpoint blockade. New England Journal of Medicine, 2018. 378(2):158-168. doi: 10.1056/NEJMra1703481

16. Russell CD, Millar JE, and Baillie JK. Clinical evidence does not support corticosteroid treatment for 2019-nCoV lung injury. The Lancet, 2020. 395(10223):473-475. doi: 10.1016/j.jtho.2020.02.025

17. Zhang $\mathrm{H}$, Huang $\mathrm{Y}$, and Xie $\mathrm{C}$. The treatment and outcome of a lung cancer patient infected with SARS-CoV-2. Journal of Thoracic Oncology, 2020. doi: 10.1016/j.jtho.2020.02.025

18. Hwang DM et al. Pulmonary pathology of severe acute respiratory syndrome in Toronto. Modern pathology, 2005. 18(1):1-10. doi.org/10.1038/ modpathol.3800247

19. Franks TJ et al. Lung pathology of severe acute respiratory syndrome (SARS): a study of 8 autopsy cases from Singapore. Human pathology, 2003. 34(8):743-748. doi.org/10.1016/S0046-8177(03)00367-8

20. Tian $S$ et al. Pulmonary pathology of early phase 2019 novel coronavirus (COVID-19) pneumonia in two patients with lung cancer. Journal of Thoracic Oncology, 2020. doi.org/10.1016/j.jtho.2020.02.010

21. Bonomi L et al. A rapid fatal evolution of Coronavirus Disease-19 (COVID-19) in an advanced lung cancer patient with a long time response to nivolumab. Journal of Thoracic Oncology, 2020. doi.org/10.1016/j.jtho.2020.03.021

22. Wu Z and McGoogan JM. Characteristics of and important lessons from the coronavirus disease 2019 (COVID-19) outbreak in China: summary of a report of 72314 cases from the Chinese Center for Disease Control and Prevention. Jama, 2020. 323(13):1239-1242. doi:10.1001/jama.2020.2648

23. Cai H. Sex difference and smoking predisposition in patients with COVID-19. The Lancet Respiratory Medicine, 2020. 8(4):e20. doi.org/10. 1016/S2213-2600(20)30117

24. Chen $\mathrm{N}$ et al. Epidemiological and clinical characteristics of 99 cases of 2019 novel coronavirus pneumonia in Wuhan, China: a descriptive study. The Lancet, 2020. 395(10223):507-513. doi.org/10.1016/S01406736(20)30211-7

25. Beaver $\mathrm{K}$ et al. Comparing hospital and telephone follow up for patients treated for stage-I endometrial cancer (ENDCAT trial): a randomised, multicentre, non inferiority trial. BJOG: An International Journal of Obstetrics \& Gynaecology, 2017. 124(1):150-160. doi.org/10.1111/14710528.14039

26. Frankland $\mathrm{J}$ et al. Follow-up care after treatment for prostate cancer evaluation of a supported self-management and remote surveillance programme. BMC cancer, 2019. 19(1):368. doi.org/10.1186/s12885-019$5561-0$

27. Schmidt-Hansen M, Baldwin DR and Hasler E. What is the most effective follow-up model for lung cancer patients? A systematic review. Journal of Thoracic Oncology, 2012. 7(5):821-824. doi.org/10.1097/ JTO.0b013e31824afc55

28. Cusack $M$ and Taylor $C$. A literature review of the potential of telephone follow up in colorectal cancer. Journal of Clinical Nursing, 2010. 19(17 18):2394-2405. doi.org/10.1111/j.1365-2702.2010.03253.

29. Pignon JP et al. Meta-analysis of chemotherapy in head and neck cancer (MACH-NC): an update on 93 randomised trials and 17,346 patients. Radiotherapy and oncology, 2009. 92(1):4-14. doi.org/10.1016/j. radonc.2009.04.014

30. Remuzzi A and Remuzzi G. COVID-19 and Italy: what next? The Lancet, 2020. doi.org/10.1016/S0140-6736(20)30627-9

31. Goel AN et al. The impact of treatment package time on survival in surgically managed head and neck cancer in the United States. Oral oncology, 2019. 88:39-48. doi.org/10.1016/j.oraloncology.2018.11.021

32. Song $S$ et al. The effect of treatment time in locally advanced cervical cance in the era of concurrent chemoradiotherapy. Cancer, 2013. 119(2):325-331. doi.org/10.1002/cncr.27652

33. Mao L et al. Neurologic manifestations of hospitalized patients with coronavirus disease 2019 in Wuhan, China. JAMA neurology, 2020 doi:10.1001/jamaneurol.2020.1127

34. Roder $\mathrm{D}$ et al. Time from diagnosis to treatment of colorectal cancer in a South Australian clinical registry cohort: how it varies and relates to survival. BMJ open, 2019. 9(9):e031421. doi: 10.1136/bmjopen-2019-031421

35. Kucejko RJ et al. How Soon Should Patients With Colon Cancer Undergo Definitive Resection? Diseases of the Colon \& Rectum, 2020. 63(2): 172182. doi: 10.1097/DCR.0000000000001525

36. Delisle $M$ et al. The Association Between Wait Times for Colorecta Cancer Treatment and Health Care Costs: A Population-Based Analysis. Diseases of the Colon \& Rectum, 2020. 63(2):160-171. doi: 10.1097/ DCR. 0000000000001517

37. Siegel RL, Miller KD and Jemal A. Cancer statistics, 2019. CA: a cancer journal for clinicians, 2019. 69(1):7-34. doi.org/10.3322/caac.21551

38. Mehta $P$ et al. Correspondence COVID-19: consider cytokine storm syndromes and. Lancet, 2020. 6736(20):19-20. doi. org/10. 1016/S01406736(20)30628.

39. Shewan, L.G., A.J.S. Coats, and M. Henein. Authors' Responsibilities and Ethical Publishing. in International Cardiovascular Forum Journal. 2018. DOI: 10.17987/icfj. v13i0. 525 